\title{
Cloud Computing Cost and Negotiation: A Survey
}

\author{
Jayasankar $\mathrm{R}$ \\ M.tech \\ RVCE, Bangalore
}

\author{
Poonam Ghuli \\ Assistant Professor \\ RVCE, Bangalore
}

\begin{abstract}
Cloud computing brings advancement in the field of information technology. Cloud computing now offers organizations more choices regarding how to run infrastructures, save costs, and delegate liabilities to third party providers. It offers variety of computing services to end users. These services are offered at different prices using various pricing schemes and techniques. End clients will support the service supplier offering the best service with the most minimal cost. Cloud pricing models are complicated, which makes purchasing decisions for consumers difficult. Comparing of prices across providers is a challenge as well. This survey paper is based on the various proposed cost models and already executed cost models. This paper attempts to provide ideas that may help to build more appropriate pricing models in future that will benefit consumers as well as service providers.
\end{abstract}

\section{Keywords}

Cloud Computing, Pricing, Negotiation, service models

\section{INTRODUCTION}

Cloud Computing is a model for enabling convenient, ondemand network access to a shared pool of configurable computing resources (e.g., networks, servers, storage, applications, and services) that can be rapidly provisioned and released with minimal management effort or service provider interaction. Many other definitions are provided in [1,2,3]. Cloud computing has get to be utilized more to the purpose of being a productive business. The suppliers have begun to charge clients for what they utilize. In any case, the pricing model accessible today in cloud computing does not support compromise. This will help to create more benefit as well as to build the clients' fulfilment on the grounds that they will have a say in it.. The supplier's fulfilment comprises in boosting the benefit and usage, while the purchaser's fulfilment is in light of the cost going to be paid.

There are growing numbers of articles related to cloud computing in libraries. Chudnov described his personal experience of using cloud services Amazon EC2 and S3 in an

informal tone, costing him 50 cents[4]. Jordan discussed OCLC's strategies of building its next generation of services in cloud and provided a clear view of OCLC's future directions for us[4]. Mitchell in [4] wrote two articles: one was to make a case for using the cloud while the other provided more details of moving a library's IT infrastructure (ILS, website, and digital library systems) to a cloud along with discussing motivation, results, and evaluation in three areas (quality and stability, impact on library services, and cost) On the cost discussion, Mitchell mentioned the difficulty of calculating technology Total Cost of Ownership (TCO) and cited two papers suggesting minimal cost savings. Mitchell suggested the same but did not provide detailed cost information. Mirsa and Mondal proposed a suitability index and a Return on Investment (ROI) model by taking into consideration impacts and real value. Their suitability index and ROI model is well thought but considers using the cloud for every aspect of all IT operations as a whole.

Cloud computing can be deployed within an enterprise, as a private cloud, since it is restricted in access to the enterprise's private network. In contrast, a service provider can provide cloud services to its customers in a public cloud. An environment that transparently combines multiple clouds, private or public, is known as a hybrid cloud. NIST describes three service model layered [5] based on computing architecture.

Software as a Service (SaaS) allows users to use the cloud computing providers' applications through a thin client interface such as a web browser. In the SaaS model, the cloud computing providers manage almost everything in the cloud infrastructure (e.g., physical servers,network, OS, applications). It is directly targeted for general end users. The end users can directly run applications on the clouds and do not need install, upgrade,and backup applications and their work. Typical SaaS products are Google Apps and Salesforce Sales CRM.

Platform as a Service (PaaS) allows users to deploy their own applications on the provider's cloud infrastructure under the provider's environment such as programming languages, libraries, and tools. In this model, the cloud computing providers manage everything except the application in the cloud infrastructure. PaaS is directly targeted for general software developers. They can develop, test, and run their codes on a PaaS platform.Typical examples of this model includes Google AppEngine,Windows Azure, and Joyent.

Infrastructure as a Service (IaaS) allows users to manage processing, storage, networks, and other fundamental computing resources so that they can deploy and run arbitrary software such as operatingsystems and applications.In this model, the providers only manage underlying physical cloud infrastructure (e.g. physical servers and network), and provides services via virtualization. The users have maximum control on the infrastructure as if they own underlying physical servers and network. Leading providers of this model includes Amazon, VMware, Linode, Rackspace, Joyent, and IBM Blue Cloud.

Table 1:Different vendors and the services provided by them

\begin{tabular}{|l|l|}
\hline Cloud Computing Provider & Layer \\
\hline Akamai & PaaS, SaaS \\
\hline Amazon Web Services & IaaS, PaaS, SaaS \\
\hline EMC & SaaS \\
\hline Eucalyptus & IaaS open source software \\
\hline
\end{tabular}




\begin{tabular}{|l|l|}
\hline Google & PaaS(AppEngine), SaaS \\
\hline IBM & PaaS, SaaS \\
\hline Linode & IaaS \\
\hline Microsoft & PaaS (Azure), SaaS \\
\hline Rackspace & IaaS, PaaS, SaaS \\
\hline Salesforce.com & PaaS, SaaS \\
\hline VMware vCloud & PaaS, IaaS \\
\hline Zoho & SaaS \\
\hline
\end{tabular}

\section{CLOUD COMPUTING PRICING SCHEMES}

Most interesting factor is the way vendors charge consumers for the resources requested. Few providers offer hourly (usage-dependent), component-based rates. Some try to attract customers by a low price per GB of storage while charging hidden costs for inbound and/or outbound data transfer or even for data transfer within the provider's infrastructure. A closer look at the different types of Clouds shows that for the usage of services from a Public Cloud the service provider delivers the necessary resources. These resources are also chargeable. Virtual machines, server blade, network services, security services, service-level agreements, data services and WAN services are some of the examples of chargeable resources.

A deeper survey into the pricing schemes reveals, pricing model for cloud providers can be either static or dynamic. In static modeling, as the name indicates, the prices are fixed allowing the vendors to collect the revenue based on per time interval used, hence achieves better scalability. The user has to pay for the resources used. Resources can be either different types of data structures active in query execution or one of the above mentioned resources. On the other hand, providers use dynamic pricing for federated clouds, where resources are shared among many cloud service providers. In dynamic pricing, price is set based on a number of factors such as availability, time, etc. For example, a user requesting an instance at peak time will pay less due to the system being fully utilized. If the system has less users then the price is going to be high. One example of dynamic pricing is spot instance from Amazon.

Fixed prices were easier to understand and more straightforward for users. However, fixed pricing could not be fair to all users because not all users had the same needs. Hence, a study proposed charging variable prices with advanced reservation [6][7]. Charging variable pricing with advanced reservation would let users know the exact expenses that are computed at the time of reservation even though they were based on variable prices. The paper emphasized the importance of implementing autonomic metered pricing to increase revenues. Autonomic metered pricing can also be straightforward for users through the use of advanced reservations. The advantage of advanced reservations is that users can not only know the prices of their required resources in the future but are also able to guarantee access to future resources to better plan and manage their operations.

The leaders of cloud computing are continuously implementing new ideas to make pricing more attractive to

customers which helps them to overcome their competitors. For example Microsoft Azure has introduced the pricing per minute to match the prices per hour provided by Amazon. Building a pricing plan requires associating costs to pricing for billable items and then combining these into different plans that are offered to cloud consumers. Each billable item can have different pricing options that can be either fixed or variable pricing. In fact, many providers offer different pricing options for same billable item. Hence, following are the several pricing options.

Fixed Recurring Pricing: Fixed recurring plan is the simplest pricing option, where providers offer a fixed periodic cost for the billable item. For example, a virtual machine can be offered at a fixed cost per month. The consumer is billed the same amount every month without the consideration of actual usage. Fixed recurring pricing can also be used as a base price for a billable item

Variable Pricing by Resource Consumption(Pay per Use)[8]: Variable pricing by resource consumption involves billing the consumer for the actual amount of atomic units of the billable item that were used during a specific period, where atomic unit is the smallest possible unit of measurement and collection, for a billable item, that will be used for billing purposes.. For example, an IaaS deployment might measure the CPU usage of virtual machines and calculate pricing based on the consumed amount of processing power measured in $1 \mathrm{GHz}$ units.

Variable Pricing by Time: Variable pricing by time involves billing the consumer based on how long the billable item was used. The units here are modeled as time increments. For example, the price can be modeled per hour of usage. For a virtual machine priced on an hourly basis, the consumer can be billed monthly for the total number of hours the virtual machine was in use (powered on) in that month.

Cost Multipliers: Some billable resources might be modeled as cost multipliers, rather than giving a standalone price. For example, data services such as compression and encryption can be modeled as multipliers on the cost of storage. SLAs can be priced in a similar manner.

\section{ASSUMPTIONS AND APPLIED COST STRUCTURE}

This section compares the pricing schemes mentioned above and some of the instances of pricing models offered by leading cloud providers such as Amazon and Microsoft Azure. Several other pricing schemes have been proposed based on the analysis of historical utilization ratio of the resources, current prices, availability of resources for the next round, and final price. IT services that are delivered as cloud services typically have the following characteristics described.

The most commonly implemented pricing model amongst others is pay-as-you-go model, with minimal costs. Here users can request for as many resources as they want without having any long-term commitment. For example, Microsoft currently offers pricing on per minute basis. This model comes under fixed pricing. Hence, it is considered as unfair to the customers because he might pay for more time than needed, as payment is done on periodic basis.

Advantages: Customer is aware of the exact price to be paid, since resources are reserved for the customer, for the requested period of time.

Disadvantages: Service provider may reserve the resources for longer than what has customer requested. Since the price 
is fixed service provider cannot increase or decrease the price based on the demand. The other most commonly implemented pricing model is Subscription model (static or fixed). As the name indicates, here price is based on the period of subscription. The major drawback of this model is customer might underpay for the resources if he uses it extensively, causing problems to the provider. If the usage of resources is less, customer might end up paying extra price than required.

Financial economic model for dynamic pricing, here pricing is based on the usage of resources. This model is considered as fair for both the service provider and customer, since price is set based the resource utilization and it offers a high level of QoS to customers. Value-based pricing is another commonly implemented dynamic pricing model where price is set based the value perceived by the customer.

\section{CLOUD COMPUTING NEGOTIATION}

Service Level Agreement (SLA), an essential part of cloud computing describes the negotiation between the provider and customer regarding the services provided. Negotiation is a critical factor since it affects customer behavior, loyalty to the provider and the organization's success. An SLA might involve agreements between the cloud provider and customer regarding QoS, pricing, guarantees, and so on. However, negotiation is founded on the following three main attributes: price, utilization and availability of resources. This section provides an overview about some negotiation mechanism and strategies.

Lot of companies are acquiring different negotiation processes[9] in order to thrive. Companies are adopting attractive negotiation techniques because it is considered as the key point for business survival, growth and empowerment. It is seen as time spent to make profit and cost effective relationships with other companies that will potentially promote the negotiators reputation. If a company decides to go for a negotiation model, they need to know the key elements for negotiation process:

- Making a deal through a final offer from a buyer or seller.

- It needs to make sure there are participants such as customers interested in offer.

- Advertising the best offers and messages send between the participants to come up with a deal suitable for both sides.

- Send back messages between the participants when the deal changes.

Market leaders use a very simple negotiation protocol, in which the customer is presented some offers from which he can either chose to accept or reject them or send back a new proposal. Time constraints are specified prior to agreement, since this can go on for months. Few of various negotiation strategies are: Contract Net, Auction Model, Game Theory Based and Discrete Optimal Control Model. These strategies are selected based on the type of business each provider is running. Provider needs to make an elaborate analysis of the negotiation mechanism selected to make sure he/she has selected a proper model and is suitable for the business, so that it can bring benefits on the long run.

Contract Net: This protocol is used to exchange SLA between the provider and the customer. In contract Net task announcements are evaluated by an agent, who acts as manager and bids for the tasks to other agents. Contract Net is suitable for multilateral processes, since this type of protocol has only two possible outcomes: accept or reject. The drawback of this protocol is it is not useful for bilateral process. Customers' claim that the protocol cannot provide an acceptable feedback for negotiated agreement between the two parties. So most of the firms do not use this protocol. Since the goal of using negotiation, mechanism is to bring benefit to both the parties through communication and interaction.

Auction Model: Auction model is the most commonly used negotiation mechanism. It is used for dynamic resource allocation. Auctions are generally one-to-many negotiations, a seller can have many buyers or vice versa. Both the parties agree on a proposed pricing scheme without any penalties in case of rejection by any of the parties. The different types of negotiations that come under auction model are [9]: Firstprice sealed, bid auction, Vickrey auction, Electronic bulletin board approach, multi-round sealed bid auction, Yankee auction and many more.

Game Theory Based Model: In this model, each agent is given the responsibility to offer a plan, the negotiation is terminated if the offered plan is accepted by all the users. This plan is not used by many firms though.

Discrete Optimal Control Plan: This model is optimized for market use and has been adapted by many leading firms. The model consists of three steps: gathering all the required information, coming up with a decision and making the decision known. The information acquired can be in terms of resources required, availability, QoS, price, tasks and the number of tasks etc. After gathering all the information, an agent can decide an appropriate algorithm to come up with an optimal solution.

Negotiation can have many attributes to negotiate over, however the mechanisms described above in this dissertation mainly concentrate on price.

\section{CONCLUSION}

In this paper, we have discussed the key concepts of cloud computing, pricing schemes used by service providers to charge customers and different negotiation models. Customers can chose the pricing model based the resources required. Resources can be in terms of virtual machines, QoS, availability, end user utility, user satisfaction etc. The customer can chose the service providers with the pricing scheme that is most compatible with the customer's behavior. For example, a customer who needs to use the cloud extensively would be better off using the services of a cloud with a static pricing approach than a dynamic one because a static approach would charge less in this case. Customers with limited usage would fare better if they used the services of a cloud with a dynamic pricing approach.

In conclusion, negotiation models can definitely have an impact on the cloud computing business. It can maximize the profit as well the utilization of resources.

\section{REFERENCES}

[1] "Cloud Computing and Grid Computing 360-Degree compared", I. Foster, I. Yong, Z .Raicu and S. Lu,IEEE Grid Computing Environments Workshop ,pp-1-10, 2008.

[2] ,"A Break in the Clouds", Towards a cloud Definition,Comput.Commun.Review, L. M. Vaquero, L 
Rodero-Merino, J. Caceres and M. Lindner ACM SIGCOMM vol. 39, no. 1,(2009).

[3] "Above the Clouds: A Berkeley View of Cloud Computing", M. Armbrust, A. Fox, R. Griffith, A. D. Joseph, R. Katz, A. Konwinski, G. Lee, D. Patterson, A. Rabkin, I. Stoica and M. Zaharia,Publication of Reliable Adaptive Distributed Systems Laboratory, Berkeley ,(2009).

[4] Cloud Computing: Case Studies and Total Costs of Ownership Benedikt Martens, Marc Walterbusch and Frank Teuteberg, 2012 45th Hawaii International Conference on System Sciences.

[5] The Cloud Computing : A Systematic Review,Mohammad
Oqail Ahmad, Dr.Rafiqul Zaman Khan, IJIRCCE,may 2015

[6] Cloud Computing Pricing Models: A Survey,May Al,Roomi, Shaikha AlEbrahim, Sabika Buqrais and Imtiaz Ahmad,International Journal of Grid and Distributed Computing,2013.

[7] "Cloud Computing and its pricing scheme", Varun Kamra, Kapil SonaWane, Pankaja Alappanavar, 2012.

[8] "Managing the Real Cost of On-Demand Enterprise Cloud Services with Chargeback Models".white paper by ciscosystems,2010 Cisco Systems

[9] "Investigating Pricing and Negotiation Models for Cloud Computin"g Raluca Suzana Andra, August , 2013,EPCC 